\title{
Assessment of renal volume by 3D VOCAL Ultrasonography method in late-onset growth- -restricted fetuses with normal amniotic fluid index
}

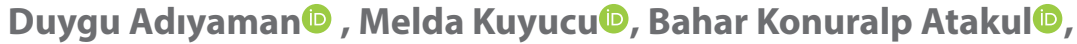 \\ Hakan Gölbaşı(D, Halil Gürsoy Pala@ \\ Tepecik Training and Research Hospital, Department of Obstetrics and Gynecology, Division of Perinatology, Izmir, Turkey
}

\begin{abstract}
Objectives: The aim of this study was to study renal volumetric alterations and renal artery doppler changes in late-onset fetal growth restricted (FGR) fetuses with normal amniotic fluid compared to healthy pregnancies.

Material and methods: This prospective study was composed of pregnant women with late-onset FGR and a control group of uncomplicated pregnancies within 32-37 weeks of gestation. Following the assessment of umbilical, bilateral uterine, middle cerebral using Doppler Ultrasonography (US), three dimensional (3D) US Virtual Organ Computer-aided Analysis (VOCAL) was executed to calculate bilateral renal volumes.

Results: A total of 76 fetuses with FGR and 51 healthy fetuses (control group) were evaluated. Umbilical artery Doppler systole/diastole and Pulsatility index values were found to be significantly different between the two groups $(p=0.001$ and $p=0.001$, respectively). Middle cerebral, bilateral uterine, and bilateral renal arteries' Doppler indices revealed no difference between the two groups. Right, left, and mean renal volume of the fetuses with FGR were smaller than the control group, and the differences were statistically significant $(p=0.025, p=0.004, p=0.004$, respectively). Left renal volume was significantly greater than the right renal volume in the control group $(p=0.009)$.

Conclusion: Although not accompanied by oligohydramnios, and having similar renal vascular resistance as the control group, renal volumes of fetuses with late-onset FGR were still observed lower than the control group. This difference was explained by not decreased blood flow via redistribution but other mechanisms like glomeruli reduction and glomerular apoptosis.
\end{abstract}

Key words: late-onset fetal growth restriction; 3D VOCAL Ultrasonography; renal volume

Ginekologia Polska 2020; 91, 11: 679-684

\section{INTRODUCTION}

Fetal growth restriction (FGR) affects approximately 10 percent of all pregnancies. Compared to healthy pregnancies, FGR has been associated with higher perinatal mortality and morbidity and may require more prenatal care [1].

Intrauterine hypoxemia, mostly due to placental insufficiency, causes remodeling of the fetal circulation. Because of this remodeling event, the fetal hemodynamic profile tends to distribute low oxygenated fetal blood towards to the vital organs, including the brain, myocardium, and adrenals, and a reduction in blood supplied to kidneys, fetal intestines, and lungs [2].

Interestingly, kidneys are not considered as vital organs in the intrauterine period. Contrarily, lower renal perfusion due to FGR has been related to life-time complications in adulthood, including coronary heart disease, stroke, hyper- tension, type 2 diabetes, and chronic kidney disease that can manifest later in adulthood [2-4].

Nephrogenesis is thought to be continued even after birth, although most of the nephrons are resolved within the intrauterine period. An adverse intrauterine environment, such as intrauterine growth restriction, causes sclerosis in multiple organ systems, including fetal kidneys [5]. Because of this sclerosis, oligonephropathy has been associated with future renal hypertension and proteinuria $[2,6,7]$. In previous studies, a decreased number of glomeruli and lower renal volume in pregnancies with FGR have been widely discussed in all aspects. However, prenatal renal changes have not thoroughly researched $[2,3,5]$.

According to several doppler studies, renal arteries have been shown to have higher resistance and pulsatility indexes (PI) in fetuses with FGR. On the other hand, this difference 
can be more noticeable when FGR is accompanied by oligohydramnios $[8,9]$. According to literature, hypoperfusion due to FGR tends to not affect renal artery Doppler PI values unless oligohydramnios has been detected, which can be explained by the redistribution process [9].

Late-onset FGR is defined as growth restriction that is diagnosed following 32 weeks of pregnancy. It is not associated with the reduced villous vascular area of the placenta like early-onset FGR and is not always associated with abnormal Doppler parameters and cardiovascular adaptations. Although cardiovascular changes are not always accompanying, oligohydramnios due to hypoperfusion of fetal kidney is believed to be a complication of late-onset FGR [10].

Fetal kidney volumes are expected to be smaller in fetuses with FGR, and this difference is usually explained by the lack of renal artery blood flow [6]. Oligohydramnios due to decreased renal arterial flow is the major complication of fetuses with FGR. However, we hypothesize that when oligohydramnios is not accompanying late-onset $F G R$, renal volumes of fetuses with FGR may not differ from healthy pregnancies.

\section{Objectives}

The aim of this study was to present the renal volumetric alterations, and renal artery Doppler changes in intrauterine growth-restricted fetuses with normal amniotic fluid indexes compared to healthy pregnancies. We hypothesize that, compared to healthy pregnancies, pregnancies with late-onset FGR have similar renal volumes and renal Doppler indexes when oligohydramnios is not present.

\section{MATERIAL AND METHODS}

\section{Patients and data collection}

This prospective study was conducted between March 2019 to December 2019 at Tepecik Training and Research Hospital, Department of Obstetrics and Gynecology, Division of Perinatology. The same institution's ethics committee approved the study. One hundred twenty-seven pregnant women between 32-37 weeks of gestation were enrolled in this study following provided written informed consent. The data on age, parity, body mass index (BMI), and smoking preference were collected, along with a detailed ultrasound scan. Women with singleton pregnancies were included, and the gestational age was calculated after confirming with the first trimester crown-rump length. All of the enrolled pregnancies were previously scanned in their second trimester and had no significant structural or chromosomal abnormalities. None of the pregnant women had a history of chronic diseases such as diabetes, hypertension, systemic lupus erythematosus, and antiphospholipid syndrome that could have led to placental vascular damage. Cases with proven FGR causes, such as preeclampsia and gestational hypertension, and pregnancies complicated by polyhydramnios were also excluded. Finally, the birth week, weight, mode of delivery, and Apgar scores were collected.

Pregnants assessed as FGR with normal amniotic fluid index after 32 weeks of gestation (late-onset FGR) were enrolled in this study. Oligohydramnios due to hypoperfusion of fetal kidney is believed to be a complication of FGR. However, FGR with oligohydramnios was not the interest of this study.

\section{MATERIAL AND METHODS Scanning}

One sonographer with minimum of 6 years of experience made all examinations (HGP). The ultrasound machine was a Samsung Ultrasound System HS70A (Samsung Medison Company, Republic of Korea). Measurements were performed during limited fetal movement or respiration. No pressure with the ultrasound probe was applied to the fetus. All biometric measurements and amniotic fluid index calculations were performed using the $2 \mathrm{D}$ probe. Estimated fetal weight (EFW) was calculated by four-way biometric measurements (biparietal diameter, head circumference, abdominal circumference, and femur length) using Hadlock's formula [11]. Umbilical artery (UA), middle cerebral artery (MCA), and uterine artery Doppler assessments were made by techniques defined by the ISUOG Practice Guideline [12]. Pregnancies assessed FGR who have EFW of less than the $3^{\text {rd }}$ percentile or EFW of less than the $10^{\text {th }}$ percentile with an abnormal UA Doppler or an abnormal cerebroplacental ratio (CPR) according to Delphi criteria [13]. Abnormal UA is accepted as UA pulsatility index (PI) of greater than the $95^{\text {th }}$ percentile or with absent or reversed UA diastolic flow. Abnormal CPR was accepted as the CPR of less than the $5^{\text {th }}$ percentile Pregnancies complicated with FGR after 32 weeks of gestation was defined as late-onset FGR. Amniotic fluid index (AFI) was computed by measuring amniotic fluid in four quadrants. Amniotic pockets without cord and extremities were measured, and AFI less than $50 \mathrm{~mm}$ were interpreted as oligohydramnios [14]. Renal abnormalities and renal pelvis diameters more than $10 \mathrm{~mm}$ were excluded [15].

After presenting kidneys properly in the coronal axis, we performed 3D scanning with a 3D/4D curved array abdominal probe. The image was magnified, and kidneys were placed into the 3D volume box. In the coronal view, six consecutive images with a rotation angle of $30^{\circ}$ were captured, two demarking arrows placed, and manual tracing was applied to all of the images. The volumetric calculation was conducted automatically by VOCAL software (Fig. 1).

\section{Statistics}

Statistical analysis was performed with IBM SPSS Statistics 25.0 package program (IBM Corp., Armonk, New York, 


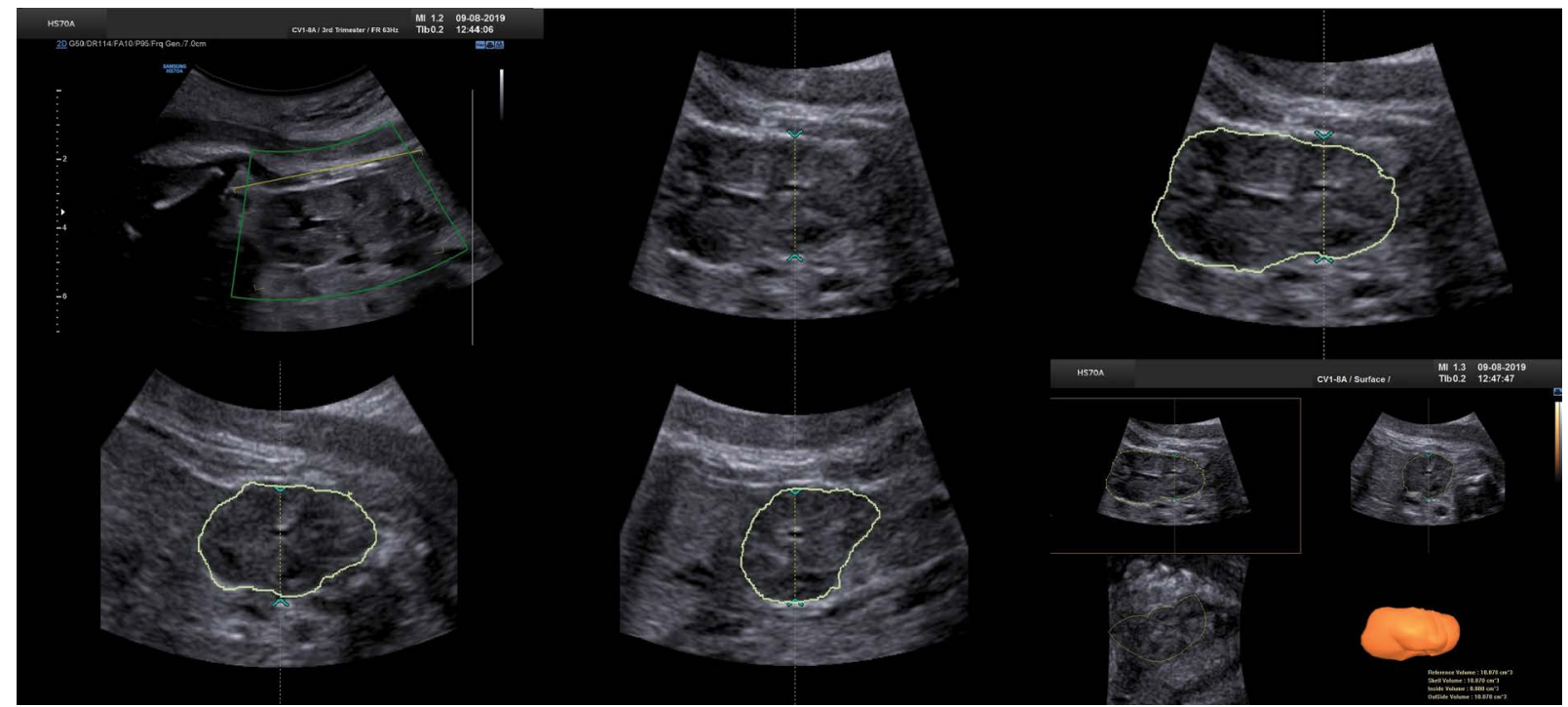

Figure 1. Volumetric calculation of fetal kidney by virtual organ computer-aided analysis (VOCAL) software

Table 1. Maternal, perinatal characteristics of the control group and FGR group $(n=127)$

\begin{tabular}{|l|l|l|l|}
\hline Characteristics & $\begin{array}{l}\text { Control } \\
(\mathbf{n = 5 1 )}\end{array}$ & $\begin{array}{l}\text { FGR } \\
\text { (n=76) }\end{array}$ & p value \\
\hline Maternal age & $26(10)$ & $27.5(11)$ & $2(1)$ \\
\hline Gravida & $2(2)$ & $1(1)$ & $p>0.05^{\ddagger}$ \\
\hline Parity & $1(2)$ & $27.3(6.5)$ & $p>0.05^{\ddagger}$ \\
\hline BMI $\left[\mathrm{kg} / \mathrm{m}^{2}\right]$ & $28(8.4)$ & $35(2)$ & $p>0.05^{\ddagger}$ \\
\hline $\begin{array}{l}\text { Gestational age } \\
\text { at exam [weeks] }\end{array}$ & $34(3)$ & $10(13.2 \%)$ & $p>0.05^{\ddagger}$ \\
\hline Smoking & $5(9.8 \%)$ & $p>0.05^{\dagger}$ \\
\hline
\end{tabular}

FGR — Fetal growth restriction; BMI — Body mass index; Data are presented as Median and interquartile range or $n(\%) ; \ddagger$ Mann-Whitney test; $\uparrow$ Chi-square Test

USA). Shapiro-Wilk's test, a histogram, and $Q-Q$ plot was used to assess the normality of data of this study. After defining the normality, means and standard deviations or medians and interquartile range (IQR) were provided for continuous variables, whereas frequencies and percentages were provided for categorical variables. The Chi-Square statistic was performed for testing categorical variables. Independent sample t-test for parametric and Mann-Whitney $U$ test for non-parametric variables was used to distinguish the differences between FGR and the control groups. The volume difference between the left and right kidney were tested by paired sample t-test. A p-value of less than 0.05 was regarded as statistically significant.

\section{RESULTS}

One hundred forty-three singleton pregnancy were enrolled in this study. Unfortunately, we could not manage to assess fetal renal volume (right, left, or both) due to inappropriate fetal position and/or movement in 16 pregnancies. A total of 76 fetuses with FGR and 51 fetuses with normal estimated fetal weight were studied. The mean gestational age was 34 weeks in the control group and 35 weeks in the FGR group. There was no statistically significant difference between groups in terms of maternal and perinatal characteristics $(p>0.05)$. The characteristics of the subjects are given in Table 1. There was a statistically significant difference between the right and left renal volume. The left renal volume was greater than the right one when both FGR and control groups were included. $(p=0.009)$. However, this difference was not prominent in the FGR group, and no statistical significance was observed within this group $(p=0.15)$. However, in controls, the left renal kidney was larger than the right kidney $(p=0.02)$ (Tab. 2).

UA Doppler studies revealed that there was a statistically significant difference between the two groups. As expected, FGR had higher S/D (S/D (Systole/Diastole) and PI values $p=0.00$ and $p=0.001$, respectively. MCA Doppler studies were not different between groups. Likewise, there was no significant difference between groups in uterine artery S/D and PI. Right and left renal arteries S/D values 
Table 2. Difference between right and left renal volume in all pregnancies

\begin{tabular}{|l|l|l|l|l|l|l|l|}
\hline Measurement & Mean \pm SD & SEM & Mean Difference & SE Difference & t & df & p value \\
\hline $\begin{array}{l}\text { Control } \\
\text { right renal volume }\end{array}$ & $10.41 \pm 2.56$ & 0.21 & & & & \\
\hline $\begin{array}{l}\text { Control } \\
\text { left renal volume }\end{array}$ & $11.19 \pm 2.68$ & 0.24 & 0.78 & 0.32 & 2.37 & 50 & 0.021 \\
\hline $\begin{array}{l}\text { FGR } \\
\text { Right renal volume }\end{array}$ & $9.43 \pm 2.26$ & 0.21 & & & & & \\
\hline $\begin{array}{l}\text { FGR } \\
\text { Left renal volume }\end{array}$ & $9.78 \pm 2.61$ & 0.24 & 0.35 & 0.24 & 75 & 0.15 \\
\hline
\end{tabular}

FGR — Fetal growth restriction; Data are presented as mean and standard deviation. Paired sample t-test was used

\begin{tabular}{|c|c|c|c|}
\hline & Control $(n=51)$ & FGR $(n=76)$ & $p$ value \\
\hline UAS/D & $2.39(0.65)$ & $2.63(0.96)$ & $0.000^{\ddagger}$ \\
\hline UA PI & $0.87(0.3)$ & $0.98(0.32)$ & $0.001^{\ddagger}$ \\
\hline MCA S/D & $4.39(1.75)$ & $4.73(1.94)$ & $0.46^{\ddagger}$ \\
\hline MCA PI & $1.56(0.49)$ & $1.7(0.59)$ & $0.64^{\ddagger}$ \\
\hline Right uterine artery PI & $2.07(0.53)$ & $2.14(0.78)$ & $0.81^{\ddagger}$ \\
\hline Left uterine artery PI & $0.84(0.33)$ & $0.85(0.45)$ & $0.72^{\ddagger}$ \\
\hline Right renal artery S/D & $5.60(256)$ & $5.53(1.91)$ & $0.67^{\ddagger}$ \\
\hline Left renal artery S/D & $5.43(1.86)$ & $5.46(2.47)$ & $0.25^{\ddagger}$ \\
\hline Right renal artery PI & $1.99 \pm 0.39$ & $2.08 \pm 0.58$ & $0.28^{\S}$ \\
\hline Left renal artery PI & $2.04(0.64)$ & $2.01(0.56)$ & $0.76^{\ddagger}$ \\
\hline Right renal volume $\mathrm{cm}^{3}$ & $10.41 \pm 2.56$ & $9.43 \pm 2.26$ & $0.025^{\S}$ \\
\hline Left renal volume $\mathrm{cm}^{3}$ & $11.19 \pm 2.68$ & $9.78 \pm 2.61$ & $0.004^{\S}$ \\
\hline Mean renal volume $\mathrm{cm}^{3}$ & $10.80 \pm 2.34$ & $9.60 \pm 2.20$ & $0.004^{\S}$ \\
\hline $\mathrm{EFW}[\mathrm{gr}]$ & $2611(572)$ & $2029(38)$ & $0.000^{\ddagger}$ \\
\hline EFW percentile & $47(38)$ & $3.43(4.47)$ & $0.000^{\ddagger}$ \\
\hline Birth week & $38 \pm 1.80$ & $36.91 \pm 1.4$ & $0.002^{\S}$ \\
\hline Birth weight [gr] & $3100(730)$ & $2282(580)$ & $0.000^{\ddagger}$ \\
\hline Apgar score $0^{\text {th }}$ minute & $7.35 \pm 0.87$ & $7.26 \pm 1.06$ & $0.66^{\S}$ \\
\hline Apgar score $5^{\text {th }}$ minute & $8.32 \pm 0.90$ & $8.31 \pm 1.12$ & $0.95^{\S}$ \\
\hline Patients delivered in our center & $31(60.8 \%)$ & $58(76.3 \%)$ & \multirow{2}{*}{$0.094^{\dagger}$} \\
\hline Patients delivered in another center & $20(39.2 \%)$ & $18(23.7 \%)$ & \\
\hline Vaginal delivery & $10(32.3 \%)$ & $20(34.5 \%)$ & \multirow{2}{*}{$1.00^{\dagger}$} \\
\hline C-section & $21(67.7 \%)$ & $38(65.5 \%)$ & \\
\hline
\end{tabular}

UA — umbilical artery; MCA — middle cerebral artery; S/D — Systole/Diastole; PI — pulsatility index; EFW — estimated fetal weight; Data are presented as median and interquartile range or mean and standard deviation or $\mathrm{n}(\%) ;{ }^{\ddagger}$ Mann-Whitney test; ${ }^{\S}$ Independent sample test; ${ }^{\dagger}$ Chi-square Test

were not different statistically significant $(p=0.67$ and $p=0.25$, respectively). PI values of right and left renal arteries were also indifferent $(p=0.28$ and $p=0.76$, respectively)

Right, left, and mean renal volume of fetuses with FGR was less than the control group, and this difference was statistically significant $(p=0.025, p=0.004, p=0.004$, respectively) (Tab. 3). EFW, delivery methods, Apgar scores, and perinatal outcomes were listed in Table 3.

\section{DISCUSSION}

FGR is thought to be one of the most complicated issues of perinatal medicine, and it is also considered to have effects that may manifest later in adulthood. Barker [16] hypothesized that FGR could be related to future coronary heart disease, stroke, hypertension, and diabetes. It stated that intrauterine environmental conditions could be related to altered developmental mechanisms that have long term consequences. 
FGR is mostly caused by placental villous vascular damage and results in the activation of several compensatory mechanisms, including redistribution of fetal blood circulation towards the vital organs such as the fetal brain, adrenals, coronaries. In the fetal brain, middle cerebral arteries tend to have lower circulatory resistance when blood flow alteration mechanisms are active. In our study, no statistically significant difference between the two groups was observed for fetal MCA S/D or PI values. This result can be interpreted as our FGR group was not exposed to the brain sparing effect or redistribution due to FGR, and it is known that late-onset FGR does not always associate with the deprived blood supply of fetal organs $[17,18]$.

As compared to the brain, fetal renal arteries tend to have higher circulatory resistance to maintain the hemodynamic well-being of the fetus [19]. Vyas S et al. [19] stated that the renal artery $\mathrm{PI}$ is expected to be higher in fetuses with FGR; however, our data indicated otherwise. We did not find a significant difference between the FGR and uncomplicated pregnancies right and left renal arteries S/D and $\mathrm{PI}$ values, which is consistent with previous studies about late-onset FGR [6]. This difference might be explained by the fact that we have excluded fetuses with FGR accompanied by oligohydramnios. Oligohydramnios is proven to be secondary to decreased renal blood flow [20]. With the findings of our study, it can be commented that late-onset FGR without decreased renal flow, redistribution of the circulation is not the only mechanism in determining the fetal kidney volumes. Other mechanisms like glomeruli reduction and apoptosis may also be responsible for the decreased renal volume [7, 21, 22]. Low nephron number and small filtration area may be the leading cause of decreased renal volume than decreased renal vascular flow [23]. It is expected that MCA PI values tend to be different between the two groups, but there was no statistical difference [22].

No difference was observed between the uterine arteries of the FGR and control group on both the right and left sides. Early-onset FGR is considered to be related to higher uterine artery S/D and PI values [10, 24]. As mentioned before, our study group consisted of late-onset FGR, which uterine artery Doppler assessment typically remains normal. Therefore, a lack of a significant difference between the two groups was not suprising [25].

While most researchers in literature used 2D US in order to calculate the renal volumes of the FGR or normal pregnancies, to the best of our knowledge, this is the first study that concentrated on volume computing with the $3 D$ VOCAL method [6]. Although there are controversial data about the volumetric organ calculations with the $2 \mathrm{D}$ US compared to the 3D VOCAL technique [4, 20, 26], 2D US has more commonly been used in organ volume calculations. However, the 3D VOCAL method is thought to be more accurate in organs with non-geometric shapes such as kidneys. Additionally, calculations can be done simultaneously in about three minutes with the VOCAL method, while the 2D US calculations should be made using a formula for ellipsoid shapes, even though kidneys are not ellipsoid [27]. Our control group renal volumes were consistent with calculations of 2D US; nonetheless, the data were limited [28]. Further studies are needed on the compatibility of two methods. We have conducted research with healthy pregnancies because of insufficient data with 3D VOCAL method.

In literature, some studies suggest that there was no significant difference between the right and left renal volume $[26,28,29]$. However, according to our data, left renal volume was significantly greater than the right renal volume in the control group), but there were no differences between the two sides of renal volumes in fetuses with FGR. The number of our control group was 51, and this difference can be explained by a lack of subjects. Only, Shi et al. [30] mentioned to have found the left renal volume greater than the right one but in pediatric patients.

We should mention the limitations of this study. We conducted the study with a single operator, ignoring the interobserver reliability. Authors are now carrying out another study about the interobserver variability of organ volumes in the 3D US VOCAL method. Secondly, we have only concentrated on fetuses between 32-37 weeks and predominantly late-onset FGR. Fetuses with early-onset FGR is known to be generally exposed to intrauterine hypoxia longer; therefore, struggling more for supplying organ systems [31]. Lastly, our mean EFW percentile was 3.43 (IQR 4.47), and we accepted fetuses with FGR when EFW was less than ten percentile. Although it is obvious that our FGR group seemed to be formed by growth-restricted fetuses, they can be small for gestational age fetuses inside the FGR group. However, all of them had an abnormal UA Doppler.

\section{CONCLUSIONS}

Our findings suggest that not only renal vascular adaptations, as well as glomerulosclerosis or apoptosis, may be responsible for the decreased renal volume in fetuses with FGR.

\section{Ethical statements}

All procedures followed were in accordance with the ethical standards of the responsible committee on human experimentation (institutional and national) and with the Helsinki Declaration of 1964 and later versions.

\section{Financial Disclosure}

There are no financial conflicts of interest to disclose. 


\section{REFERENCES}

1. Thompson JL, Kuller JA, Rhee EH. Antenatal surveillance of fetal growth restriction. Obstet Gynecol Surv. 2012; 67(9): 554-565, doi: 10.1097/OGX.0b013e31826a5c6f, indexed in Pubmed: 22990459.

2. Visentin S, Grumolato F, Nardelli GB, et al. Early origins of adult disease: low birth weight and vascular remodeling. Atherosclerosis. 2014; 237(2): 391-399, doi: 10.1016/j.atherosclerosis.2014.09.027, indexed in Pubmed: 25463063.

3. Schreuder MF, Nyengaard JR, Fodor $M$, et al. Glomerular number and function are influenced by spontaneous and induced low birth weight in rats. J Am Soc Nephrol. 2005; 16(10): 2913-2919, doi: 10.1681/ASN.2004100875, indexed in Pubmed: 16093454.

4. Chang $\mathrm{CH}$, Tsai PY, Yu CH, et al. Predicting fetal growth restriction with renal volume using 3-D ultrasound: efficacy evaluation. Ultrasound Med Biol. 2008; 34(4): 533-537, doi: 10.1016/j.ultrasmedbio.2007.10.006, indexed in Pubmed: 18055097.

5. Rodríguez MM, Gómez $\mathrm{AH}, \mathrm{Abitbol} \mathrm{CL}$, et al. Histomorphometric analysis of postnatal glomerulogenesis in extremely preterm infants. Pediatr Dev Pathol. 2004; 7(1): 17-25, doi: 10.1007/s10024-003-3029-2, indexed in Pubmed: 15255031.

6. Silver LE, Decamps PJ, Korst LM, et al. Intrauterine growth restriction is accompanied by decreased renal volume in the human fetus. Am J Obstet Gynecol. 2003; 188(5): 1320-1325, doi: 10.1067/mob.2003.270, indexed in Pubmed: 12748506.

7. Bassan H, Trejo LL, Kariv N, et al. Experimental intrauterine growth retardation alters renal development. Pediatr Nephrol. 2000; 15(3-4): 192-195, doi: 10.1007/s004670000457, indexed in Pubmed: 11149109.

8. Mari G, Kirshon B, Abuhamad A. Fetal renal artery flow velocity waveforms in normal pregnancies and pregnancies complicated by polyhydramnios and oligohydramnios. Obstet Gynecol. 1993; 81(4): 560-564, indexed in Pubmed: 8459967.

9. Stigter RH, Mulder EJ, Bruinse HW, et al. Doppler studies on the fetal renal artery in the severely growth-restricted fetus. Ultrasound Obstet Gynecol. 2001; 18(2): 141-145, doi: 10.1046/j.1469-0705.2001.00493.x, indexed in Pubmed: 11529994.

10. Figueras F, Caradeux J, Crispi F, et al. Diagnosis and surveillance of late-onset fetal growth restriction. Am J Obstet Gynecol. 2018; 218(2S):S790-S802.e1, doi: 10.1016/j.ajog.2017.12.003, indexed in Pubmed: 29422212.

11. Hadlock FP, Harrist RB, Sharman RS, et al. Estimation of fetal weight with the use of head, body, and femur measurements--a prospective study. Am J Obstet Gynecol. 1985; 151(3): 333-337, doi: 10.1016/00029378(85)90298-4, indexed in Pubmed: 3881966.

12. Bhide $A$, Acharya $G$, Bilardo CM, et al. ISUOG practice guidelines: use of Doppler ultrasonography in obstetrics. Ultrasound Obstet Gynecol. 2013; 41(2): 233-239, doi: 10.1002/uog.12371, indexed in Pubmed: 23371348.

13. Gordijn SJ, Beune IM, Thilaganathan B, et al. Consensus definition of fetal growth restriction: a Delphi procedure. Ultrasound Obstet Gynecol. 2016; 48(3): 333-339, doi: 10.1002/uog. 15884, indexed in Pubmed: 26909664.

14. Reddy UM, Abuhamad AZ, Levine D, et al. Fetal Imaging Workshop Invited Participants. Fetal imaging: executive summary of a joint Eunice Kennedy Shriver National Institute of Child Health and Human Development, Society for Maternal-Fetal Medicine, American Institute of Ultrasound in Medicine, American College of Obstetricians and Gynecologists, American College of Radiology, Society for Pediatric Radiology, and Society of Radiologists in Ultrasound Fetal Imaging workshop. Obstet Gynecol. 2014; 123(5): 1070-1082, doi: 10.1097/AOG.0000000000000245, indexed in Pubmed: 24785860.

15. Odibo AO, Marchiano D, Quinones JN, et al. Mild pyelectasis: evaluating the relationship between gestational age and renal pelvic anterior-posterior diameter. Prenat Diagn. 2003; 23(10): 824-827, doi: 10.1002/pd.709, indexed in Pubmed: 14558027.
16. Barker DJP. The developmental origins of chronic adult disease. Acta Paediatr Suppl. 2004; 93(446): 26-33, doi: 10.1111/j.1651-2227.2004. tb00236.x, indexed in Pubmed: 15702667.

17. Hernandez-Andrade E, Stampalija T, Figueras F. Cerebral blood flow studies in the diagnosis and management of intrauterine growth restriction. Curr Opin Obstet Gynecol. 2013; 25(2): 138-144, doi: 10.1097/GCO.0b013e32835e0e9c, indexed in Pubmed: 23354073.

18. Rizzo G, Mappa I, Bitsadze V, et al. Role of Doppler ultrasound at time of diagnosis of late-onset fetal growth restriction in predicting adverse perinatal outcome: prospective cohort study. Ultrasound Obstet Gynecol. 2020; 55(6): 793-798, doi: 10.1002/uog.20406, indexed in Pubmed: 31343783.

19. Vyas S, Nicolaides KH, Campbell S. Renal artery flow-velocity waveforms in normal and hypoxemic fetuses. Am J Obstet Gynecol. 1989; 161(1): 168-172, doi: 10.1016/0002-9378(89)90257-3, indexed in Pubmed: 2665495.

20. Seravalli V, Miller JL, Block-Abraham D, et al. The Relationship between the Fetal Volume-Corrected Renal Artery Pulsatility Index and Amniotic Fluid Volume. Fetal Diagn Ther. 2019; 46(2): 97-102, doi: 10.1159/000491749, indexed in Pubmed: 30300895.

21. Schreuder M, Delemarre-van de Waal H, van Wijk A. Consequences of intrauterine growth restriction for the kidney. Kidney Blood Press Res. 2006; 29(2): 108-125, doi: 10.1159/000094538, indexed in Pubmed: 16837795.

22. Verburg BO, Geelhoed JJM, Steegers EAP, et al. Fetal kidney volume and its association with growth and blood flow in fetal life: The Generation R Study. Kidney Int. 2007; 72(6): 754-761, doi: 10.1038/sj.ki.5002420, indexed in Pubmed: 17637711.

23. Keijzer-Veen $M G$, Kleinveld $H A$, Lequin $M H$, et al. Renal function and size at young adult age after intrauterine growth restriction and very premature birth. Am J Kidney Dis. 2007; 50(4): 542-551, doi: 10.1053/j. ajkd.2007.06.015, indexed in Pubmed: 17900453.

24. Soregaroli M, Valcamonico A, Scalvi L, et al. Late normalisation of uterine artery velocimetry in high risk pregnancy. Eur J Obstet Gynecol Reprod Biol. 2001; 95(1): 42-45, doi: 10.1016/s0301-2115(00)00358-4, indexed in Pubmed: 11267718.

25. Committee Opinion No. 640: Cell-free DNA Screening for Fetal Aneuploidy. Obstet Gynecol. 2015 [Epub ahead of print]; 126(3)-692, doi: 10.1097/AOG.0000000000001007, indexed in Pubmed: 26114726.

26. Tedesco GD, Bussamra LC, Araujo Júnior E, et al. Reference range of fetal renal volume by three-dimensional ultrasonography using the VOCAL method. Fetal Diagn Ther. 2009; 25(4): 385-391, doi: 10.1159/000236151, indexed in Pubmed: 19786784.

27. Geelhoed JJ, Taal HR, Steegers EAP, et al. Kidney growth curves in healthy children from the third trimester of pregnancy until the age of two years. The Generation R Study. Pediatr Nephrol. 2010; 25(2): 289-298, doi: 10.1007/s00467-009-1335-2, indexed in Pubmed: 19898876.

28. Barbosa RM, Souza RT, Silveira C, et al. Reference ranges for ultrasound measurements of fetal kidneys in a cohort of low-risk pregnant women. Arch Gynecol Obstet. 2019; 299(2): 585-591, doi: 10.1007/s00404-0185032-x, indexed in Pubmed: 30607595.

29. Yu C, Chang C, Chang F, et al. Fetal renal volume in normal gestation: a three-dimensional ultrasound study. Ultrasound Med Biol. 2000; 26(8): 1253-1256, doi: 10.1016/s0301-5629(00)00298-2, indexed in Pubmed: 11120361

30. Shi Bo, Yang Yi, Li SX, et al. Ultrasonographic renal volume in Chinese children: Results of 1683 cases. J Pediatr Surg. 2015; 50(11): 1914-1918, doi: 10.1016/j.jpedsurg.2015.05.011, indexed in Pubmed: 26117811.

31. Dall'Asta A, BrunelliV, Prefumo F, et al. Early onset fetal growth restriction. Matern Health Neonatol Perinatol. 2017; 3: 2, doi: 10.1186/s40748-0160041-x, indexed in Pubmed: 28116113. 